\title{
Inhibition Mechanism Research about CD59 Specific Site Closed Short Peptide on T Cell Leukemia
}

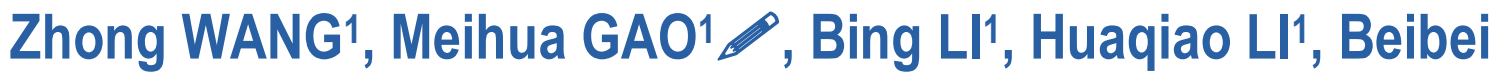 CONG11, Yi WANG11, Fengyu ZHANG'1, Le XIONG1}

\author{
${ }^{1}$ Department of Immunology ,Basic Medical College of Qingdao University, Qingdao Shandong Province, CHINA
}

\begin{abstract}
Objective: To study SP22 the closed short peptide of CD59 specific site on Jurkat cell signal transduction molecules (LAT, STAT3) genes, proliferation genes (Nf-kb3, Bcl-2) and apoptosis genes (caspase-3) effect, research CD59 specific site closed by short peptide concrete mechanism of Jurkat cells. Methods: Jurkat cells were divided into blank control group and SP22 peptide closed group, Training after 48h respectively with the method of CCK 8 to detect each cell proliferation rate; Flow cytometry -- check each group cell apoptosis; Real time quantitative PCR --from gene level determination of two kinds of cells before and after the experiment the expression of stat 3 and joint protein Lat, detection of cell proliferation and apoptosis protein before and after test the $\mathrm{Nf}-\mathrm{kb} 3, \mathrm{Bcl}-2$, the expression of Caspas-3; Enzyme-linked immunosorbent assay (ELISA) to detect the related cytokines. Results: add SP22 peptide group cell proliferation inhibition rate is greater than the blank control group $(\mathrm{P}<0.01)$;Add SP22 group compared with blank control group Lat, Stat3, Nf-kb3, Bcl-2 gene expression decline, Caspas-3 gene expression rise; Add SP22 $40 \mathrm{mg}$ group cell death ratio increased, compared with the blank control group difference has statistically significant $(\mathrm{P}<0.01)$. In short peptide SP22 group, cells secrete and promote tumor to growth of interleukin 2 (IL-2) and TGF- $\beta$ had been inhibited. Conclusion: CD59 specific site closed short peptide can inhibit Jurkat cell proliferation, reduce the expression of Lat, Stat3, Nf-kb and Bcl-2, activating caspase-3, promote the apoptosis of Jurkat cells.
\end{abstract}

Keywords: CD59, Close peptideSP22, Jurkat cell, Apoptosis

\section{INTRODUCTION}

CD59 is widely distributed in hematopoietic cells and the surface of hematopoietic cells and tissue cells, by GPI anchored on the cell membrane, is one of important regulatory proteins in the end of the complement activation pathways. CD59's main function is to inhibit the formation of complement attack membrane complex, protect the host cell dissolution from complement mediated. In addition, CD59 is also involved in cell adhesion and signaling transduction, by tyrosine kinase activation of neutrophils, inhibiting tumor cell apoptosis, participate in tumor metastasis, new blood vessel formation and tissue regeneration [1], and can be used as biomarkers of cancer stem cells [2]. Tumor cell membrane on the surface of high expression of CD59 molecules, making 
tumor cells to evade the immune system attack by complement mediated and in tumor cells of drug resistance phenomenon, it is an important factor that the failure of tumor treatment in wide variety clinical patients. Theoretically reduce the expression of CD59 on the tumor cells membrane or decline the CD59 activity can be reversed escape of the immune. But the active site of research with complement bond reports about CD59 molecules is very little at home and abroad. Our research group designed and synthesized short peptide closed CD59 active site bond with complement using phage peptide library screening technology ${ }^{[3]}$.The purpose of this paper adopt specific short peptide to close complement combine with CD59 active site to act on Jurkat cells, discusses it's specific mechanism to promote Jurkat cells apoptosis.

\section{$1 \quad$ Materials and methods}

1.1 Materials Jurkat cells kept by our laboratory; RPMI 1640 culture

medium bought from HyCLone company;CD59 specific site closed short peptide (1 $000 \mathrm{mg} / \mathrm{L}$ ) designed by our research group, synthesized from suzhou Qiangyao biological technology co., LTD.; CCK kit bought from Beijing Fanbo biological chemical co., LTD.; Stat3, Lat, Nf-кb3, Bcl-2, Caspas-3 gene primers synthesized from Huada genomic biotechnology co., Apoptosis Kit Annexin V Apoptosis Detection Kit purchased from eBioscience co., reverse transcription Kit, Faststart Greeen Master Essential DNA fluorescence quantitative kit bought from Roche co., ELISA

kit bought from Abcam co.

\subsection{Methods}

1.2.1 Cell culture in contains $100 \mathrm{ml} / \mathrm{L}$ fetal bovine serum, 10mg/L penicillin and streptomycin RPMI 1640 medium, $37^{\circ} \mathrm{C}, 50 \mathrm{ml} / \mathrm{L} \mathrm{CO} 2$ incubator in cultured cells, every $48 \mathrm{~h}$ change liquid in batches, adopt the logarithmic phase cell used experiment.

1.2.2 CCK8 test the cells proliferation and inhibition adopt CCK8 measurement to observe the CD59 specific site closed short peptide effect on Jurkat cells. Jurkat cells divided into blank control group and SP22 peptide group, adjust the $4 \times 10^{5}$ cells per ml, Each hole 100ul cell suspension. add 50ul 1640 liquid in each hole of blank control group ; add $10 \mathrm{mg} / \mathrm{ml}, 20$ $\mathrm{mg} / \mathrm{ml} \quad 40 \mathrm{mg} / \mathrm{ml}, \quad 80 \mathrm{mg} / \mathrm{ml}$ Four kinds of concentration short peptide 50ul in each hole of SP22 peptide group, each groups set up three holes respectively. Training after 48 hours, each hole respectively add $10 \mathrm{ul} \mathrm{CCK} 8$ reagent, continue to training 6 hours, ELISA instrument measure absorbance (OD) value at $450 \mathrm{~nm}$ wavelength.

1.2.3 Real-time quantitative PCR At the gene level determination of two groups after the experiment joint protein Lat and Stat3, cell proliferation and apoptosis gene expression of $\mathrm{Nf}-\kappa \mathrm{b} 3, \mathrm{Bcl}-2$, and Caspase -3. Cell culture after 48 hours, using TRIZOL method to extract total RNA respectively, Reverse transcription kit synthesis cDNA by reverse transcription method, according to the SYBR Premix ExTaq $^{\mathrm{TM}}$ II fluorescence quantitative kit instruction to do the real-time quantitative PCR reaction. According to PUBMED gene pool and the UNIPROT protein library to find all the mRNA coding sequences of nucleotide to design amplification primer, gene primers synthesized by Huada genomic biotechnology company. Internal reference choose housekeeping gene $\beta$-actin. Primer for upstream of Lat: 5'GGCGGGATTCTGATGGT-3’ Downstream primer: 5'- GCACCACCAGGTAGCC-3', Primers for upstream of Stat3:

5'-CAGTGACCAGGCAGAAGAT-3' Downstream primer of Stat3:

5'-TGTTGACGGGTCTGAAGTTG-3' , Primer for upstream of Nf-kb3:

5'-ACTACGACCTGAATGCTGT-3' Downstream primer of Nf-кb3:

5'-ATACACCTCAATGTCCTCTT-3', upstream Primer of

Bcl-2:

5'-GAGACAGCCAGGAGAAATCAA-3'

Downstream primer of Bcl-2: 
5'-ATGTGTGTGGAGAGCGTCAA-3', upstream

Primer of

5'-ATCCTGAGATGGGTTTATGT-3'

Primer of

Caspase-3:

Downstream

Caspase-3:

5'ATCCTGAGATGGGTTTATGT-3'. Each RNA samples detection Repeat 3 times, take 3 times the average $\mathrm{Ct}$ value of the results was analyzed $\mathrm{Ct}$ values represent each test tube fluorescent signal of required cycles to reach the setting threshold. $\Delta \mathrm{Ct}=\mathrm{Ct}$ (The purpose gene) $-\mathrm{Ct}$ (Internal reference gene), $\Delta \Delta \mathrm{Ct}$ $=\Delta \mathrm{Ct}(\mathrm{SP} 22$ group $)-\Delta \mathrm{Ct}($ control group, $2-\Delta \Delta \mathrm{Ct}$ show the Purpose gene expression difference times between the two groups .

1.2.4 Flow cytometry instrument to detect cell apoptosis: Blank control group and SP22 peptide group training after 48 hours respectively, adjust the cell density of $1 \times 10^{6} / \mathrm{ml}$. take $1 \mathrm{ml}$ respectively put in $1.5 \mathrm{ml}$ volume EP tube, wipe off supernatant after centrifugal, PBS working liquid washing one time, each tube cell suspension in 100ul Binding Buffer again, add 5ul annexin V (annexin V-APC, incubation for $15 \mathrm{~min}$ avoid light at room temperature, washing one time after centrifugal, add 200ul Binding Buffer and 5ul PI(propidium Iodide Staining Solution), Using flow cytometry instrument for testing.

1.2.5 ELISA detect the related cytokines: The blank control group add SP22 peptide group training 48 hours respectively, reserve supernatant after centrifugal, according to the ELISA kit instruction step, test IL-2 and TGF- $\beta$ respectively in the supernatant, record data, drawing standard curve of the concentration, calculating average level of two groups cytokines.

1.2.6 Statistical analysis Using statistical software SPSS17.0 analysis processing, data to show by $\mathrm{x} \pm \mathrm{s}$, the data comparison between groups adope the $t$ test methods , $\mathrm{P}<0.01$ for the difference was statistically significant.

\section{Results}

2.1 CCK8 detect proliferation and inhibition: the results confirmed that after joining peptide SP22 closed cell proliferation rate of decline, the inhibition of cell proliferation increased as the concentration of SP22 improvement, but SP22 increased to a certain concentration, the inhibition of proliferation no longer improvement (figure 1).As shown in figure 1, add SP22 each group was compared with the without SP22 $(P<$ 0.01 ) all have meaningful; $420,40,80 \mathrm{mg}$ compared respectively with $10 \mathrm{mg}$ has significance $(P<0.05)$; $\star$ show $20 \mathrm{mg}$ and $40 \mathrm{mg}$ has significant $(P<0.05)$; $40 \mathrm{mg}$ and $80 \mathrm{mg}$ has meaningless $(P>0.05)$. when the concentration of $40 \mathrm{mg}$ the inhibition of cell proliferation is strongest.

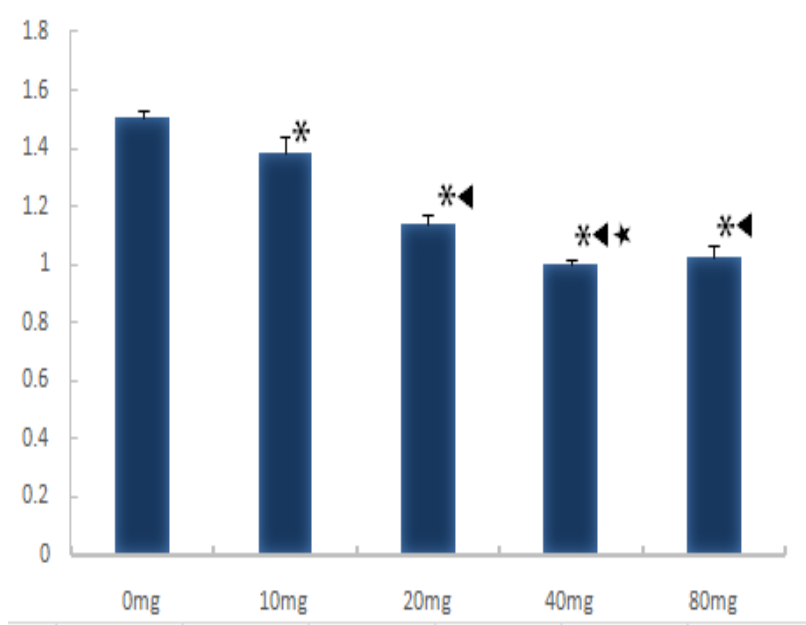

Figure 1 The proliferation rate of CCK8 testing 
2.2 Real-time quantitative PCR ---- From gene level to determine cell related protein molecule mRNA expression level: through detect the mRNA of signal transduction protein Lat, Stat3 and proliferation and apoptosis gene Bcl-2, Nf-kb3、Caspase3 by real-time quantitative PCR .Found that add SP22 group compared with blank control group Lat, , Bcl-2, Nf-кb3、Stat3 gene expression declined, but the Caspase-3 gene expression increased (table 1).

Table 1 The result of Real-time quantitative PCR detection

\begin{tabular}{ccccc}
\hline & $\begin{array}{c}\text { Blank control group } \\
(\Delta \mathrm{Ct})\end{array}$ & $\mathrm{SP} 22$ group $(\mathrm{Ct})$ & $\Delta \Delta \mathrm{Ct}$ & $2^{\wedge^{-} \Delta \Delta^{\mathrm{Ct}}}$ \\
\hline Lat & 7.08 & 11.04 & 3.96 & 0.0643 \\
\hline Nf-kb3 & 8.64 & 9.82 & 1.18 & 0.4414 \\
\hline BCL-2 & 7.45 & 8.97 & 1.52 & 0.3487 \\
\hline STAT3 & 8.60 & 9.11 & 0.51 & 0.7022 \\
\hline Caspas-3 & 8.63 & 5.64 & -2.99 & 7.9447 \\
\hline
\end{tabular}

2.3 Flow cytometry instrument to detect cell apoptosis: Results prove that add SP22 40mg group rates of cell death rising, cell death is concentrated in the upper left quadrant, compared with the blank control group the difference has statistically significant $(※ P<0.05)$, suggesting close short peptide SP22 could inhibit proliferation and promote the cell apoptosis.(figure 2)
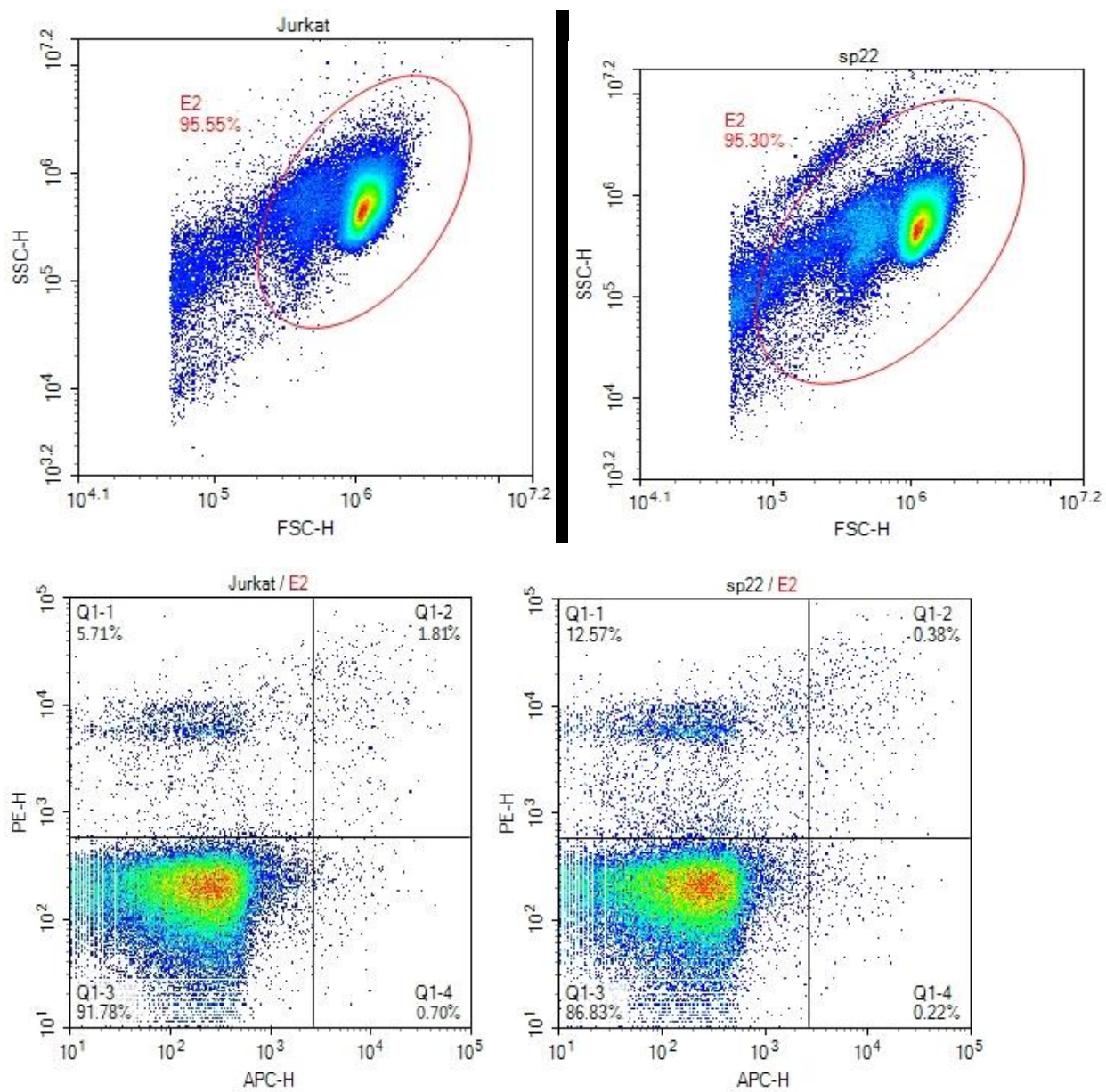


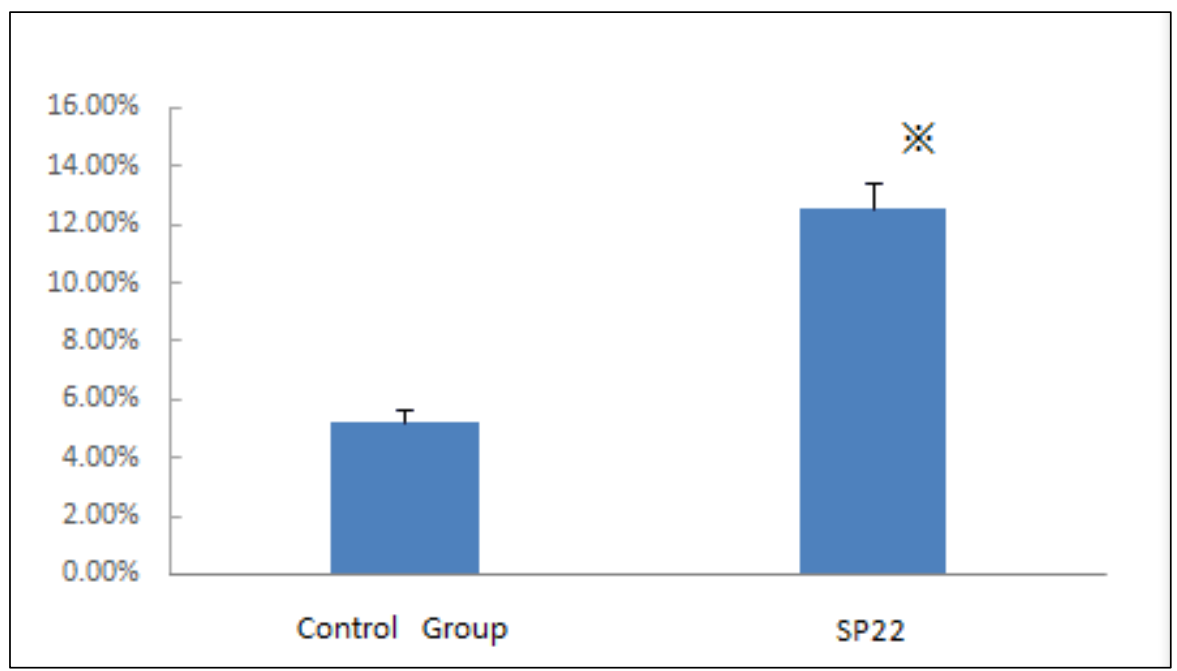

Figure 2 The result of FCM detection

2.4 ELISA detect the related cytokines Through the SP22 group and blank control group in cytokine IL-2 and TGF- $\beta$ testing, found concentration of IL-2 (figure 3) and TGF- $\beta$ (figure4) appear significantly decreased in SP2 2 group, compared with blank control group, the difference was statistically significant $(※ P<0.05)$. This show short peptide SP2 2 could inhibit cells to secrete cytokines IL- 2 and TGF- $\beta$ that promote tumor cell growth

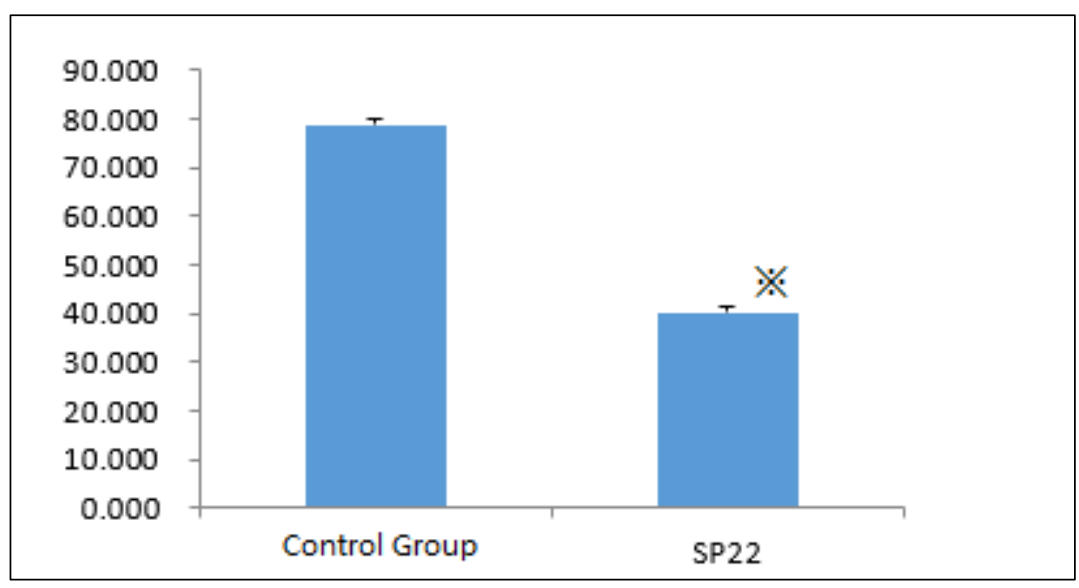

Figure 3 The level of IL-2 in ELISA testing $(※ P<0.05)$

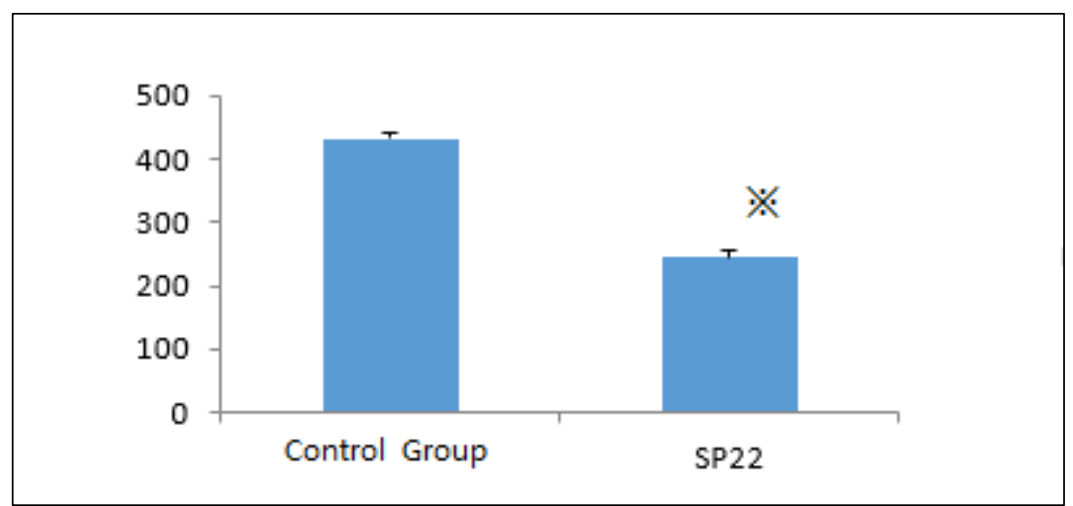

Figure 4 The level of TGF- $\beta$ in ELISA testing $(※ P<0.05)$ 


\section{Discussion}

Many studies reported in a wide variety of tumor cell membrane surface is found to have excessive expression of CD59 molecules In recent years, on the one hand, excessive expression molecules of CD59 block the attack membrane complex formation and inhibit cell dissolution from complement mediated (CDC) ${ }^{[4]}$, on the other hand also increased related signal molecule activation and express in the tumor cells proliferation signal path, the tumor cells not only escape the immune system attacks, and make the continuing growth and proliferation. So reduce the excessive expression of CD59 molecules of the tumor cells surface or interfere and closed the active site of excessive expression CD59 molecules, could be reversed the immune escape and enhanced complement mediated immune attack due to the excessive expression molecules of CD59 on tumor cells, meanwhile also can inhibit the tumor cells growth and proliferation. So closed or interference the CD59 molecules active site gradually become new hot spot in research of tumor escape and immune targeted therapy.

Our research team in the prophase experiment, using phage display technology and biological information technology design and screening short peptide sequence of amino acids that can specific combine with inhibition complement activity area of CD59 molecules, successfully designed SP22 short peptide that include 22 amino acids and can combine with specific active site of CD59. Our early experiments have confirmed that the short peptide strengthened the complement mediated immune attack after SP22 closed CD59 molecules active site, also proved through the experiment CD59 molecules shift palm acyl group to LAT, after LAT accept the palm acyl group then it can locate in the lipid rafts to play the function of signal transduction $^{[5]}$. Studies have confirmed that the expression of CD59 positive patients of paroxysmal nocturnal hemoglobinuria $(\mathrm{PNH})$ compared with the expression of CD59 negative patients $(\mathrm{PNH})$, bone marrow cells intracellular signaling pathways STAT5 protein phosphorylation level show an obvious difference $^{[6]}$ after stimulated by granulocyte colony stimulating factor (g-csf) and stem cell growth factor (C-KIT). Studies have shown CD59 molecules can still activate the signal transduction when lack expression of $\mathrm{TCR} / \mathrm{CD} 3^{[7]}$, this illustrate that CD59 molecules play important synergy in the process of signal transduction. Therefore, we speculate that not only in the presence of TCR/CD3 complex, through the non-receptor protein tyrosine kinase (nrPTK) LCK ,FYN, ZAP - 70, LAT transduce activation signal, and in the case of lack expression of TCR/CD3, CD59 can assist cytokines to transduce signal via JAK-STAT(in nrPTK) signaling pathway. Stat3 is also a key molecules in a variety of carcinogenic signaling pathways, compositional expression in tumor cells and immune cells, participate in tumor cells proliferation, differentiation, invasion, metastasis, angiogenesis and anti-apoptosis ${ }^{[8]}$. The reported is a few that CD59 molecules active site how to influence the growth of tumor cells proliferation and signal molecules transduction mechanism inside and outside of cells after closed by specific short peptide. This experiment closed CD59 molecules active site use short peptide (SP22) and detect the effect in cells signal transduction pathway. We found that SP22 group compared with blank control group, the expression of Lat, Stat3, Nf-кb3, and Bcl-2 appear an obvious decline, the difference has statistically significant $(P<0.05)$; And the expression of promote apoptosis Caspase 3 appear obvious arise, the difference has statistically significant $(P<0.05)$.

When CD59 active site is closed by short peptide (SP22), may affect the three-dimensional structure of CD59 and hampering this function that CD59 molecules to transfer palm acyl group to joint protein LAT, so the LAT downstream Nf-kb3 in signal transduction pathways has also been affected, as a

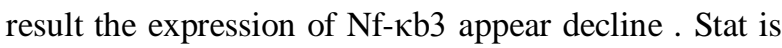
an important transduction molecules in JAK-Stat signaling pathway, mainly to accept the activation of cytokines, The most important function section of Stat is $\mathrm{SH} 2$ domain structure, it has entirely same structure 
and has the exactly same function identical $\mathrm{SH} 2$ domain "GTFLLRFSS" core sequence structure with the Src family, can recognition and combination with phosphorylation tyrosine residues on the another molecular. The expression decline reasons of Stat3: we analysis may be that CD59 molecules transfer palm acyl group to joint protein LAT was hindered after CD59 active site closed by SP22, the LAT's phosphorylation tyrosine residues identification and combination with Stat3's SH2 structural domain were also affected; also may be that the CD59 synergy was affected in the JAK-STAT signal pathway after CD59 active site closed by SP22. Declined of the Bcl-2 possible reasons: $\mathrm{Nf}-\kappa \mathrm{b} 3$ and Stat 3 can regulate and improve the expression of the Bcl-2, in this experiment Nf- $\kappa \mathrm{b} 3$ and Stat3 show decline, then the expression of Bcl-2 with decline also.

The expression arise mechanism of Caspas-3: Our research group prophase studies prove that it can inhibit the expression of survivin which is one of anti-apoptotic protein after SP22 combined with CD59 molecules on the Jurkat cell membrane, then eliminate the survivin's inhibition to the caspase- 3 protein ${ }^{[9]}$, but the mechanism of survivin how to restrained is not clear. Studies have pointed out that short peptide may be activate caspase- 3 in a similar way of TNFR1 receptor signaling pathway; However there are also documents confirm that survivin regulate the process of apoptosis no death receptor pathway involved in ${ }^{[10]}$, but regulated mainly through mitochondrial approach. Study pointed out that high expression level of Stat 3 is closely related activation with downstream target gene (Bcl-2, survivin) ${ }^{[8]}$, We analysis may be the Stat3 expression decline after add SP22, corresponding the Bcl-2 and the survivin decline too, then the inhibited effect of caspase- 3 by survivin's decline is reduced, so the expression of caspase-3 arise. Leukemia cell secretion of IL -2 in the tumor microenvironment play in the form of autocrine or paracrine effect to promote the growth of tumor cell proliferation. we found that add SP22 group compared with blank control group content of IL-2 appear obvious decline, we analysis because SP22 inhibits Jurkat cell proliferation, then cells autocrine IL-2 show decline. Tumor cells secreted TGF- $\beta$ not only promote the growth of their proliferation, can also inhibit the body's immune function. This study found that adding SP22 group contents of TGF- $\beta$ appear decrease, this show that the Jurkat cells proliferation were inhibited, so it secrete the level of TGF- $\beta$ that promote the proliferation-self declined also.

Through the above analysis, we think after CD59 active site closed by the short peptide SP22, promote apoptosis mechanism of Jurkat cell may be affected mainly by CD59-Lat and JAK-Stat3 two signaling pathway. About closed short peptide specific mechanism of action, our team will be to do further research in the next phase of the animal experiment. Our study provides a new theoretical and experimental basis for the high expression of CD59 tumor cells evade immune attack and pertinence of targeted therapy, it has important theoretical value and application prospect.

\section{References:}

1) HU Weiguo, OYANG qianwen,LUO Rongcheng. < The Roles of CD59 in the Initiation, Progression and Antibody-Based Therapy of Cancer.> [J]. Molecular imaging, 2013, 36(1): 36-39.

2) Gemei M, Di Noto R, Mirabelli P, et al. Cytometric profiling of CD133+ cells in human colon carcinoma cell lines identifies a common core phenotype and cell type-specific mosaics [J]. The International journal of biological markers, 2013, 28(3): 267-273.

3) CHENG Ying,GAO Meihua . < Specific coalescent peptide of CD59

4) screened by phage library.> [J]. Cellular and molecular immunology journal, 2006, 22(2): 164-166.

5) Gan Z, Wang B, Zhou W, et al. Molecular and functional characterization of CD59 from Nile tilapia (Oreochromis niloticus) involved in the immune response to Streptococcus agalactiae [J]. Fish \& shellfish immunology, 2015, 44(1): $50-59$.

6) GAO Meihua LI Yuanyuan CONG Beibei < GPI anchored 
protein CD59 through LAT mediated the signal transduction mechanism research of $\mathrm{T}$ cell $>[\mathrm{J}]$. Chinese journal of immunology, 2014.7, 30(874-878.

7) LIANGYong WANGQu. < STAT5 phosphorylation levels of erythropoietin and thrombopoietin receptors in $\mathrm{CD} 34^{+}$ $\mathrm{CD}^{-} 9^{-}$and $\mathrm{CD} 34^{+} \mathrm{CD} 9^{+}$bone marrow cells of patients with paroxysmal nocturnal hemoglobinuria > [J].Natl Med J China, 2011.8.16, 91(30): 2129-2131.

8) Lipp AM, Juhasz K, Paar C, et al. Lck mediates signal transmission from CD59 to the TCR/CD3 pathway in Jurkat T cells [J]. PloS one, 2014, 9(1): e85934.

9) GAO Anding LIN Baoshun. <Relationships between STAT3 and tumor> $[\mathrm{J}]$. Chinese journal of biochemistry and molecular biology 2013.5, 29(5): 397-403.

10) LI Xianping GAO Meihua. < Effect of peptide seals specific to CD59 on the expression o fapoptosis related genes in HeLa cells CD59 > $[\mathrm{J}]$. Chinese journal of Cellular and molecular immunology, 2008, 24(1): 20-26.

11) [10] CHEN Yuqing LI Wei ZHOU Jihong. < molecular mechanism of anti-apoptotic action of survivin in NCI-H446 lung cancer cells> $[\mathrm{J}]$. Chinese journal of Oncology, 2006.06, 28(6): 413-417 